\title{
Challenges in the diagnosis and management of dementia in Hong Kong
}

\author{
LW Chu *, FRCP (Lond, Edin, Glasg), FHKAM (Medicine) \\ Division of Geriatric Medicine, Department of Medicine, Li Ka Shing Faculty of Medicine, The University of Hong Kong, \\ Pokfulam, Hong Kong \\ *Iwchu@hku.hk
}

Hong Kong Med J 2017;23:218-9

DOI: 10.12809/hkmj175066

Dementia affects 47 million people worldwide. ${ }^{1}$ Persons with dementia require appropriate diagnostic investigations, treatments, and longterm care. The economic impact of dementia is huge. Globally, the total estimated worldwide cost of dementia is US\$818 billion. ${ }^{1}$ In Hong Kong, the estimated number of persons with dementia was 103433 among those aged 60 years or above in 2009 , and this number is projected to increase to 332688 by $2039 .{ }^{2}$ Dementia is a clinical syndrome due to a variety of causes. The most common is Alzheimer's disease (AD) followed by vascular dementia. ${ }^{3}$ Other causes include dementia with Lewy bodies (DLB), Parkinson's disease dementia (PDD), and frontotemporal dementia. Clinical diagnosis is often based on clinical features, with reference to the clinical criteria. ${ }^{4-7}$ Overlap of the clinical features of different dementias is common and may result in an incorrect clinical diagnosis. Notably, recent studies have confirmed the role of structural (magnetic resonance imaging $[\mathrm{MRI}])$, functional $\left(\left[{ }^{18} \mathrm{~F}\right]-2\right.$ fluoro-2-deoxy-D-glucose positron emission tomography [ $\left.{ }^{18} \mathrm{FDG}-\mathrm{PET}\right]$ or single-photon emission computed tomography [SPECT]), and amyloid pathology (carbon 11-labelled Pittsburgh compound B) brain imaging in improving the accuracy of clinical differential diagnosis of AD versus other dementia subtypes. ${ }^{4,8,9}$ For example, volumetric MRI hippocampal volumes differentiate AD from healthy elderly adults with over $80 \%$ accuracy. Recently, this has been applied in clinical practice and is preferred to the semiquantitative visual hippocampal assessment that has only $81 \%$ sensitivity and $67 \%$ specificity for AD diagnosis. ${ }^{4}$ Furthermore, new amyloid and tau PET neuroimaging for preclinical AD diagnosis will also be available soon for clinical application. ${ }^{9}$

Compared with AD, DLB and PDD are much less prevalent in Chinese than in Caucasian populations. In this issue of the Hong Kong Medical Journal, Shea et al ${ }^{10}$ reports a Chinese case series of 16 DLB and seven PDD patients from a memory clinic in Hong Kong. In contrast with the first reported series of 31 DLB and four PDD Chinese patients from Mainland China, which employed clinical assessment only, ${ }^{11}$ Shea et $\mathrm{al}^{10}$ included functional brain imaging with ${ }^{18}$ FDG-PET or technetium-99m hexamethylpropylene amine oxime SPECT in the diagnostic assessment, with hypometabolism or hypoperfusion of occipital lobes, respectively as positive evidence of DLB/PDD. With these tools, they studied the diagnostic inaccuracy of clinical assessment alone. Pre-imaging accuracy of clinical diagnosis was only $52 \%$, confirming the clinical utility of adding these imaging investigations to improve diagnostic accuracy in clinical practice. ${ }^{10}$ The spectrum of disorders with Lewy body embraces a spectrum of neurodegenerative diseases, including Parkinson's disease, PDD and DLB, that are due to the abnormal neuronal accumulation of the protein $\alpha$-synucleinopathies in the brainstem, limbic, and neocortical regions. In the diagnostic criteria, a '1year' duration between the onset of Parkinsonism and dementia symptoms is an arbitrary criterion to clinically distinguish PDD and DLB. ${ }^{9,12}$ This is being reviewed by the International Parkinson and Movement Disorder Society and may be deleted in the future. ${ }^{9}$

In elderly patients, multiple co-morbidities are common: AD may coexist with DLB and PDD in the same patient. Shea et $\mathrm{al}^{10}$ found that $52 \%$ (12 out of 23) of patients with DLB/PDD had an AD pattern of functional imaging abnormalities (ie bilateral temporoparietal lobes hypometabolism/ hypoperfusion), showing that AD actually coexisted in approximately $50 \%$ of their DLB/PDD patients. This finding also explained why $38 \%$ of them were initially diagnosed with $\mathrm{AD}{ }^{10}$ The presence of $\mathrm{AD}$ in these patients represented additional co-morbid disease and was not a 'misdiagnosis'.

Clinically, confirming the diagnosis of DLB or PDD in these patients had an important bearing on subsequent treatments. First, clinicians should avoid the use of neuroleptic drugs in these patients, owing to a high risk of neuroleptic syndrome in DLB/PDD. Second, cholinesterase inhibitors should be tried as they are beneficial in alleviating cognitive symptoms in DLB and PDD patients. Third, levodopa/carbidopa treatment of Parkinsonism motor symptoms may be complicated by a worsening of hallucinations. ${ }^{13}$ With progressive neuronal degeneration, both dementia and Parkinsonism motor symptoms will deteriorate with time. For their DLB and PDD patients, Shea et $\mathrm{al}^{10}$ reported a $30 \%$ mortality on follow-up (mean, 3.1 years), and $70 \%, 26 \%, 52 \%$, and $26 \%$ of patients had falls, pressure sores, dysphagia, and aspiration pneumonia, respectively. 
In general, most dementias are neurodegenerative in nature. The disease pathology may start in the brain 10 to 20 years before onset of dementia symptoms. With increasing dementia severity over the years, loss of self-care ability and eventually the ability to eat will occur in the final stage of the dementia illness. Feeding problems lead to weight loss, malnutrition, impaired immunity to infection, and poor wound healing. In the current issue of this Journal, Luk et $\mathrm{al}^{14}$ reviewed the clinical and ethical issues related to feeding problems in advanced dementia patients in Hong Kong. As emphasised by the authors, the key issue was the high prevalence of tube feeding: 53\% among advanced dementia persons living in old-age homes. ${ }^{14,15}$ The reasons for giving tube feeding included dysphagia, inadequate eating, and malnutrition. Tube feeding, however, did not prevent aspiration pneumonia, nor did it yield any benefit for survival. Nasogastric tube feeding also induced nasal discomfort in these demented persons and prompted attempts to selfremove the tube. The latter might lead to an increased chance of being restrained, as well as repeated hospital visits for replacement of the nasogastric tube. Some of these patients might not need a feeding tube, ${ }^{14}$ and 'careful hand feeding' could offer a viable alternative. This may work well for patients who have lost their motivation to eat but still retain their ability to swallow. Formal assessment by the speech therapist should be carried out to confirm this ability. In these patients, careful hand feeding should be tried first. A trial of antidepressants may be given if depressive mood is also present. It should be noted that careful hand feeding is not effective for advanced demented patients with genuine dysphagia in whom the risk of aspiration and aspiration pneumonia is high. Withholding feeding is another option for these patients. Obviously, the harm of withholding feeding includes dehydration, malnutrition, and eventually death. The dilemma of whether to start tube feeding or to stop feeding remains a clinical and ethical challenge for both the physician and family. In this context, the presence of an advance directive of the patient will help guide clinical treatment and care decisions. With an advance directive, a mentally competent person can indicate the form of health care he or she would like to receive in the future. In this regard, the directive must include the use or non-use of tube feeding. ${ }^{16}$ It should be noted that several local studies have previously confirmed the acceptability and feasibility of advance directives among Chinese adult and elderly patients in Hong Kong. ${ }^{17-19}$ Most demented patients, however, do not have written advance directives before becoming mentally incompetent. Our current challenge now lies in the promotion of the use of advance directives among Hong Kong citizens, while they are still mentally competent, and encouragement to formulate one.

\section{References}

1. World Alzheimer Report 2016. Improving healthcare for people living with dementia. Coverage, quality and costs now and in the future. Alzheimer's Disease International. Available from: https://www.alz.co.uk/research/ WorldAlzheimerReport2016.pdf. Accessed 10 Apr 2017.

2. Yu R, Chau PH, McGhee SM, et al. Trends in prevalence and mortality of dementia in elderly Hong Kong population: projections, disease burden, and implications for long-term care. Int J Alzheimers Dis 2012;2012:406852.

3. Chiu HF, Lam LC, Chi I, et al. Prevalence of dementia in Chinese elderly in Hong Kong. Neurology 1998;50:1002-9.

4. Chu LW. Alzheimer's disease: early diagnosis and treatment. Hong Kong Med J 2012;18:228-37.

5. McKhann GM, Knopman DS, Chertkow H, et al. The diagnosis of dementia due to Alzheimer's disease: recommendations from the National Institute on Aging-Alzheimer's Association workgroups on diagnostic guidelines for Alzheimer's disease. Alzheimers Dement 2011;7:263-9.

6. McKeith IG, Dickson DW, Lowe J, et al. Diagnosis and management of dementia with Lewy bodies: third report of the DLB Consortium. Neurology 2005;65:1863-72. Erratum in: Neurology 2005;65:1992.

7. Rascovsky K, Hodges JR, Knopman D, et al. Sensitivity of revised diagnostic criteria for the behavioural variant of frontotemporal dementia. Brain 2011;134:2456-77.

8. Shea YF, Ha J, Lee SC, Chu LW. Impact of (18)FDG PET and (11)C-PIB PET brain imaging on the diagnosis of Alzheimer's disease and other dementias in a regional memory clinic in Hong Kong. Hong Kong Med J 2016;22:327-33.

9. Saeed U, Compagnone J, Aviv RI, et al. Imaging biomarkers in Parkinson's disease and Parkinsonian syndromes: current and emerging concepts. Transl Neurodegener 2017;6:8.

10. Shea YF, Chu LW, Lee SC. A descriptive study of Lewy body dementia with functional imaging support in a Chinese population: a preliminary study. Hong Kong Med J 2017;23:222-30.

11. Han D, Wang Q, Gao Z, Chen T, Wang Z. Clinical features of dementia with lewy bodies in 35 Chinese patients. Transl Neurodegener 2014;3:1.

12. Walker Z, Possin KL, Boeve BF, Aarsland D. Lewy body dementias. Lancet 2015;386:1683-97.

13. O’Brien JT, Holmes C, Jones M, et al. Clinical practice with anti-dementia drugs: A revised (third) consensus statement from the British Association for Psychopharmacology. J Psychopharmacol 2017;31:147-68.

14. Luk JK, Chan FH, Hui E, Tse CY. The feeding paradox in advanced dementia: a local perspective. Hong Kong Med J 2017;23:306-10.

15. Luk JK, Chan WK, Ng WC, et al. Mortality and health services utilisation among older people with advanced cognitive impairment living in residential care homes. Hong Kong Med J 2013;19:518-24.

16. Chu LW. One step forward for advance directives in Hong Kong. Hong Kong Med J 2012;18:176-7.

17. Wong SY, Lo SH, Chan CH, Chui HS, Sze WK, Tung Y. Is it feasible to discuss an advance directive with a Chinese patient with advanced malignancy? A prospective cohort study. Hong Kong Med J 2012;18:178-85.

18. Ting FH, Mok E. Advance directives and life-sustaining treatment: attitudes of Hong Kong Chinese elders with chronic disease. Hong Kong Med J 2011;17:105-11.

19. Chu LW, Luk JK, Hui E, et al. Advance directive and end-oflife care preferences among Chinese nursing home residents in Hong Kong. J Am Med Dir Assoc 2011;12:143-52. 